\title{
Percepción de cambios en la práctica médica y estrategias de afrontamiento
}

\author{
M. LUZ BASCUÑÁN ${ }^{1}$, NINA HORWITZ1 ${ }^{1}$ IRENE SCHIATTINO², \\ JULIA ACUÑA ${ }^{1}$, JUAN PABLO JIMÉNEZ ${ }^{1}$
}

\section{A survey about physicians' professional satisfaction}

Background: A change in the social structure of medicine and its impact on clients has occurred in the last decades. Aim: To perform a survey about subjective wellbeing among physicians. Material and Methods: A physician's professional satisfaction survey consisting in 90 questions, was applied to 580 physicians (70\% males), working in Metropolitan Santiago. Results: Physicians perceive changes in all the examined professional domains and approximately 50\% of the changes are evaluated as negative. Change perception is a general phenomena among physicians, since there were no differences in relation to gender and only in few aspects with respect to age and medical specialty. There was a predominant positive attitude, based on the profession, to face changes. Conclusions: The positive evaluation of changes and the frequent use of managing strategies are associated with a higher satisfaction among physicians.

(Rev Med Chile 2011; 139: 1305-1312).

Key words: Data collection; Physicians; Quality of life.

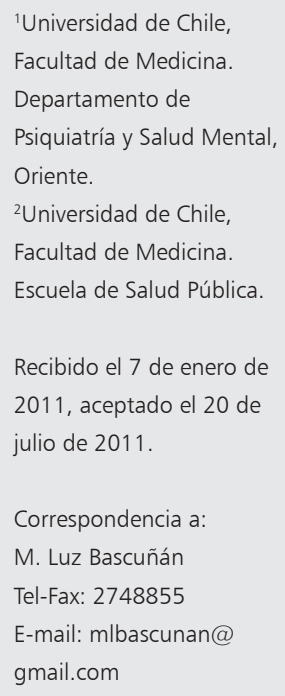

$\mathrm{N}$ umerosos trabajos han descrito importantes cambios en la estructura social de la medicina durante las últimas décadas así como su impacto en los usuarios ${ }^{1}$. Sin embargo, también existe evidencia sobre el efecto de estas transformaciones en el bienestar y satisfacción de los médicos ${ }^{2-4}$. A su vez, el bienestar de los médicos ha sido asociado a la calidad de atención y satisfacción de los pacientes ${ }^{5,6}$.

Según Rearnes y Dunstone $(1989,2001)$ la satisfacción en un momento no predice aquella posterior en el tiempo. La satisfacción se asociaría a las actitudes de los médicos ante los cambios. Cambios reportados en estos estudios incluyen la reducción de ingresos, la pérdida de control sobre las decisiones médicas y la amenaza de demandas legales ${ }^{7,8}$.

Los litigios legales serían determinantes de la satisfacción pero el efecto de tales experiencias no duraría en el tiempo9. Por su parte, el nivel de ingresos y carga laboral constituyen variables moderadoras del malestar pues su contribución para generar un mayor bienestar es limitada en comparación con otras variables como autonomía en la toma de decisiones, reconocimiento profesional, seguridad laboral, educación continua, calidad de la relación entre colegas y con los pacientes ${ }^{10,11}$.

La incorporación de controles externos ha impactado la práctica médica ${ }^{12}$. El conocimiento formal y los protocolos predefinidos toman primacía por sobre la experiencia clínica generando un nuevo profesionalismo. Harrison (2002) rotula este nuevo modelo como "medicina científica burocrática"13. La pérdida de autonomía y control sobre la profesión entonces generaría insatisfacción. Aspectos asociados son los requerimientos de las organizaciones de salud y su énfasis en los objetivos numéricos y las demandas y expectativas de los pacientes ${ }^{14}$.

Los factores descritos han sido considerados en conjunto como parte de un cambio de orden mayor en el acuerdo psicosocial tradicional entre la profesión, los empleadores, los pacientes y la sociedad ${ }^{15,16}$. Según Edwards y col (2002) mientras en el antiguo pacto existía un balance entre lo que ofrecía y recibía el médico en su trabajo, en el nuevo pacto existen imperativos adicionales sin obtener beneficios igualmente estimulantes. 
Para Ham y Alberti (2002) las bases para un nuevo pacto deben considerar intereses de todos los involucrados. Es necesario reconocer tanto los derechos de los pacientes como la responsabilidad de éstos en su salud; crear un nuevo balance entre la autoregulación y la rendición de cuentas; entre las exigencias de calidad y los recursos disponibles. Esto es concordante con una concepción del bienestar como respuesta emocional ante determinadas circunstancias. Se trataría de percepciones y sentimientos articulados en relación con el ejercicio de un rol dentro del "habitus" ${ }^{*}$ de la práctica médica actual. Tales percepciones estarían complejamente integradas con los supuestos y expectativas sobre la medicina que prevalecen en la sociedad.

Los cambios en la profesión parecen tener un efecto generalizado en el bienestar de los mé$\operatorname{dicos}^{17}$. En general, el género de los médicos no aparece como variable significativa y respecto a la edad los resultados son contradictorios ${ }^{18-20}$. Por último, tampoco existe acuerdo referente al efecto de la especialidad médica en la satisfacción ${ }^{18,21,22}$. La heterogeneidad de estos resultados puede deberse a diferencias contextuales. De allí que sea relevante distinguir los aspectos de la profesión que son examinados y revisar los hallazgos según las condiciones laborales, sistema de salud y características del contexto socio-cultural que se trate.

Este trabajo se integra en una línea de investigación iniciada el año 2004 cuyo objetivo es explorar la percepción de los médicos sobre los cambios en su profesión, las estrategias usadas ante los mismos y el impacto que los cambios tienen en su bienestar. La metodología utilizada, inspirada en el método de exploración etnográfica gradual desarrollada por la Organización Mundial de la Salud, integra métodos cualitativos y cuantitativos ${ }^{23}$. Se realizó una primera etapa cualitativa que confirmó los hallazgos internacionales en el ámbito chileno ${ }^{24-27}$. En la segunda etapa cuantitativa, se elaboró el cuestionario de bienestar subjetivo de los médicos (BISUMED) que fue validado el año 2009 en una muestra representativa de médicos colegiados de la Región Metropolitana ${ }^{28}$.

El objetivo del presente artículo es describir los resultados acerca de la percepción de cambios en la profesión, la valoración de estos cambios y las estrategias de afrontamiento ante los mismos, obtenidos en la aplicación del cuestionario BISUMED en su versión original.

\section{Material y Método}

\section{Muestra}

El universo de 6.707 médicos residentes en la Región Metropolitana inscritos en el Colegio Médico de Chile, se estratificó según las variables: género, especialidad y año de ingreso al Colegio Médico ${ }^{29}$. Se utilizó una muestra probabilística de 580 profesionales con un nivel de confianza de 95\% (Tabla 1). El promedio de edad de la muestra es de 50 años (desviación estándar de 19,9).

\section{Instrumento}

Se utilizó el cuestionario de Bienestar Subjetivo de los Médicos en su versión original ya que ésta contiene los ítems de valoración de los cambios en la medicina, centrales para nuestro objetivo que no fueron incluidos en el proceso de validación del mismo. Este instrumento está compuesto de 90 ítems divididos en dos partes. La primera contiene 12 ítems de caracterización del sujeto y la segunda 78 ítems divididos en tres secciones: 1) percepción de cambios en la profesión y su valoración; 2) estrategias de afrontamiento ante los cambios y 3) satisfacción con distintos aspectos de la vida. Este trabajo analizó: a) 16 ítems correspondientes a la percepción de cambio (escala Likert de 3 niveles: no ha habido cambios, cambios moderados, cambios profundos); b) 16 ítems de valoración de los cambios (evaluados dicotómicamente "más bien positivos" o "más bien negativos"); y c) 10 ítems de afrontamiento (escala likert de 5 niveles desde "casi nunca" a "casi siempre").

\section{Recolección y análisis de datos}

Se editó el cuestionario en agenda electrónica y en papel y se incluyó un procedimiento de consentimiento informado verbal y escrito.

Se describen los resultados para la percepción de cambios; su valoración y las estrategias de afrontamiento. Se analiza su relación con las variables género, especialidad, edad y bienestar. Para ello se aplican pruebas no paramétricas, de asociación $\left(\chi^{2}\right.$, Coeficiente de correlación de

\footnotetext{
* El concepto de habitus de acuerdo con P. Bordieu se refiere al conjunto de esquemas generativos socialmente estructurados a partir de los cuales los sujetos perciben el mundo y actúan en él.
} 
Tabla 1. Descripción de la muestra

\begin{tabular}{|llcc|}
\hline $\begin{array}{l}\text { Variables de } \\
\text { estratificación }\end{array}$ & Categorías & \multicolumn{2}{c|}{$\begin{array}{c}\text { n de casos y } \\
\text { porcentaje }\end{array}$} \\
\cline { 2 - 4 } Género & Hombre & 408 & $70,3 \%$ \\
& Mujer & 172 & $29,7 \%$ \\
& 1900 a 1975 & 177 & $30,5 \%$ \\
& 1976 a 1993 & 270 & $46,6 \%$ \\
Especialidad & 1994 a 2006 & 133 & $22,9 \%$ \\
& Pediatría & 117 & $20,2 \%$ \\
& Cirugía & 54 & $9,3 \%$ \\
& Ginecología-obstetricia & 64 & $11 \%$ \\
& Med. Interna & 79 & $13,6 \%$ \\
& Psiquiatría & 28 & $4,8 \%$ \\
& Atención primaria & 14 & $2,4 \%$ \\
& Med/Familiar & & \\
\hline Total & Otra especialidad & 224 & $38,6 \%$ \\
\hline & & 580 & $100,0 \%$ \\
\hline
\end{tabular}

Spearman), de rangos de Wilcoxon para muestras independientes y ANOVA (Kruskal Wallis).

Se consideraron las especialidades de pediatría, cirugía, ginecología-obstetricia, medicina interna; y una categoría que integra las otras especialidades. Para evaluar el bienestar se consideró el factor "bienestar general" derivado del proceso de validación del instrumento BISUMED que explicó la mayor proporción de la varianza. Este incluye 11 ítems de satisfacción con la vida, logro de metas y desarrollo personal y profesional ${ }^{29}$.

\section{Resultados}

\section{Percepción de cambios en la profesión}

Como puede observarse en la Tabla 2, existe una proporción significativamente

Tabla 2. Percepción de cambios en los aspectos examinados

\begin{tabular}{|c|c|c|}
\hline Aspecto de cambio examinado & $\begin{array}{c}\text { No ha habido } \\
\text { cambios } \\
(\%)\end{array}$ & $\begin{array}{c}\text { Ha habido cambios } \\
\text { moderados o } \\
\text { profundos }(\%) * *\end{array}$ \\
\hline Nivel de desarrollo tecnológico & 2,9 & 97,1 \\
\hline Nivel de autonomía y control del médico sobre su profesión & 15,9 & 83,6 \\
\hline Acceso del médico a la información y tecnología & 2,3 & 97,7 \\
\hline Introducción de aspectos administrativos y de gestión & 12,8 & 86,7 \\
\hline Nivel de exigencia y expectativas de los pacientes & 2,1 & 97,9 \\
\hline Nivel de información de los pacientes & 3,4 & 96,5 \\
\hline Nivel de confianza mutua en la relación médico-paciente & 14,2 & 85,7 \\
\hline Nivel de intervención de profesionales no médicos & 11,7 & 88,1 \\
\hline Introducción de variables legales en la profesión & 5,9 & 93,4 \\
\hline Relación trabajo-ingreso & 18,1 & 81,6 \\
\hline Prestigio social del médico & 13,2 & 86,8 \\
\hline Calidad de la relación entre colegas & 36,3 & 63,8 \\
\hline Presencia de médicos extranjeros en su lugar de trabajo & 36,3 & 62 \\
\hline Priorización por AUGE en la atención de pacientes & 26,3 & 70,2 \\
\hline Introducción de protocolos y guías clínicas basadas en la evidencia (AUGE) & 20,7 & 76,2 \\
\hline $\begin{array}{l}\text { Responsabilidad institucional por la defensa de los médicos frente a proble- } \\
\text { mas médicos legales (AUGE) }\end{array}$ & 41,7 & 55,7 \\
\hline
\end{tabular}

**Diferencia significativa en todos los aspectos evaluados $p<0,05$. Los porcentajes que no resultan en un $100 \%$ se debe a que no se respondió la pregunta. 
mayor de médicos que percibe cambios (moderados o profundos) que aquellos que no perciben cambios, en todos los ámbitos de la profesión examinados.

La percepción de cambios no difiere según el género. La edad mostró diferencias significativas en: la introducción de aspectos administrativos en la profesión ( $\mathrm{p}=0,001)$, intervención de profesionales no médicos $(\mathrm{p}=0,002)$ y responsabilidad institucional ante problemas legales $(0,002)$. En todos los casos aquellos de mayor edad perciben cambios con una frecuencia significativamente mayor que los de menor edad.

La especialidad se asoció con la percepción de cambios en el prestigio social $(p=0,024)$ y la presencia de médicos extranjeros en el trabajo $(\mathrm{p}=0,001)$. En todas las especialidades existe una mayor proporción de médicos que percibe cambios en el prestigio social, sin embargo, en medicina interna esta proporción es significativamente menor. En relación a la presencia de médicos extranjeros sólo entre los pediatras existe una proporción mayor de médicos que no percibe cambios.

La percepción de cambios se asocia al bienestar en tres aspectos: acceso a la información y tecnología $(\mathrm{p}=0,003)$, prestigio social $(\mathrm{p}<0,001)$ y priorización por AUGE en la atención $(\mathrm{p}=0,002)$. Presentan mayor bienestar quienes perciben cambios en el acceso a la información y tecnología; y quienes no perciben cambios en el prestigio social ni priorización por AUGE en la atención de pacientes.

\section{Valoración de los cambios percibidos}

Como se aprecia en la Tabla 3, existen diferencias significativas en la valoración de 12 de los 16 aspectos de cambio examinados y tienden a ser evaluados negativamente 9 de ellos.

La valoración de los cambios se asocia al género sólo en el prestigio social donde las mujeres tienden a evaluarlo negativamente en una proporción aun mayor que los hombres $(\mathrm{p}=0,031)$.

Respecto la edad, los médicos mayores valoran

Tabla 3. Valoración de los cambios percibidos

\begin{tabular}{|lcc|}
\hline Aspecto de cambio examinado & $\begin{array}{c}\text { Positivamente } \\
\text { valorados (\%) }\end{array}$ & $\begin{array}{c}\text { Negativamente } \\
\text { valorados (\%) }\end{array}$ \\
\hline Nivel de desarrollo tecnológico & 96,9 & $3,1^{*}$ \\
\hline Nivel de autonomía y control en el ejercicio de la profesión & 31,3 & $68,1^{*}$ \\
\hline Acceso del médico a la información y tecnología & 98,5 & $1,5^{*}$ \\
\hline Introducción de aspectos administrativos y de gestión en la práctica médica & 59,6 & $40,1^{*}$ \\
\hline Nivel de exigencia y expectativas de los pacientes & 56,6 & 43 \\
\hline Nivel de información de los pacientes & 80,6 & $19,1^{*}$ \\
\hline Nivel de confianza mutua en la relación médico-paciente & 35,5 & $64,4^{*}$ \\
\hline El nivel de intervención de profesionales no médicos & 41,5 & $58,2^{*}$ \\
\hline Introducción de variables legales en la profesión & 17,6 & $81,7^{*}$ \\
\hline Relación trabajo-ingreso & 16,5 & $83,1^{*}$ \\
\hline Prestigio social del médico & 9,8 & $89,7^{*}$ \\
\hline Calidad de la relación entre colegas & 24 & $766^{*}$ \\
\hline Presencia de médicos extranjeros en su lugar de trabajo & 47,3 & 49,1 \\
\hline Priorización por AUGE en la atención de pacientes & 47,7 & 47,3 \\
\hline Introducción de protocolos y guías clínicas basadas en la evidencia (auge) & 69,4 & $27^{*}$ \\
\hline Responsabilidad institucional por la defensa de los médicos frente a problemas & 38,6 & $57,3^{*}$ \\
médicos legales & & \\
\hline
\end{tabular}

*Diferencia significativa entre valoración positiva y negativa, $p<0,05$. 
negativamente con mayor frecuencia los cambios en el nivel de autonomía $(\mathrm{p}=0,03)$, de exigencia y expectativas de pacientes $(\mathrm{p}<0,001)$, de confianza en la relación médico-paciente $(\mathrm{p}=0,02)$, en el prestigio social $(\mathrm{p}<0,001)$, la priorización por AUGE $(p=0,02)$ y la responsabilidad institucional frente a problemas legales $(\mathrm{p}<0,001)$.

La especialidad se asocia sólo a la valoración de los cambios en el nivel de exigencia y expectativas de pacientes $(\mathrm{p}=0,007)$ y la presencia de profesionales no médicos $(\mathrm{p}=0,044)$. Una mayor proporción de médicos de especialidades primarias valora positivamente los cambios en el nivel de exigencia de los pacientes mientras que la mayoría de aquellos de "otras especialidades" los valora negativamente. Los cambios en la presencia de profesionales no médicos en el trabajo, son valorados positiva y negativamente en proporciones semejantes excepto en ginecología-obstetricia donde se valoran negativamente en una proporción significativamente mayor.

Quienes valoran positivamente los cambios refieren también mayor bienestar. Esto se manifiesta en la valoración de los siguientes aspectos : nivel de autonomía $(\mathrm{p}<0,001)$, introducción de aspectos administrativos $(\mathrm{p}=0,002)$, exigencias y expectativas de pacientes $(\mathrm{p}<0,001)$, confianza en la relación médico-paciente $(\mathrm{p}<0,001)$, intervención de profesionales no médicos $(p=0,001)$, introducción de variables legales $(\mathrm{p}=0,003)$, relación trabajo-ingreso $(\mathrm{p}<0,001)$, prestigio social $(\mathrm{p}<0,001)$, calidad de la relación entre colegas $(\mathrm{p}<0,001)$, presencia de médicos extranjeros en el lugar de trabajo ( $\mathrm{p}=0,001)$, priorización por AUGE en la atención $(\mathrm{p}=0,002)$ y responsabilidad institucional frente a problemas legales $(\mathrm{p}=0,001)$.

\section{Estrategias de afrontamiento ante los cambios}

Las estrategias para enfrenar los cambios tienden a buscarse dentro de la profesión. El 70,3\% de los médicos indicó que ante los cambios en la profesión "casi siempre" intenta rescatar lo que considera esencial de la medicina y sus buenas prácticas y $64,2 \%$ "casi siempre” intenta mejorar sus capacidades con el estudio y perfeccionamiento. Como se observa en la Tabla 4, considerando las dos puntuaciones mayores, estos porcentajes aumentan a 95,5\% y 90,8\% respectivamente.

Estas estrategias tienden a ser individuales o asociadas al grupo de trabajo cercano más que

Tabla 4. Porcentaje de respuesta para tipos de estrategias de afrontamiento ante los cambios en la profesión entre quienes respondieron

\begin{tabular}{|lccc|}
\hline Frente a los cambios & $\begin{array}{c}\text { Casi nunca/ } \\
\text { rara vez }\end{array}$ & $\begin{array}{c}\text { Algunas } \\
\text { veces }\end{array}$ & $\begin{array}{c}\text { Frecuentemente/ } \\
\text { casi siempre }\end{array}$ \\
\hline $\begin{array}{l}\text { Busco nuevas herramientas y enfoques para entender lo que pasa } \\
\begin{array}{l}\text { Intento rescatar lo que considero esencial de la medicina y sus } \\
\text { buenas prácticas }\end{array}\end{array}$ & 4,9 & 14 & $81,2^{*}$ \\
\hline $\begin{array}{l}\text { Intento mejorar mis capacidades a través del estudio y perfeccio- } \\
\text { namiento }\end{array}$ & 0,1 & 3,8 & $95,5^{*}$ \\
\hline $\begin{array}{l}\text { Amplio mis actividades profesionales (docencia, investigación, } \\
\text { gestión) }\end{array}$ & 21,5 & 17,7 & $90,8^{*}$ \\
\hline $\begin{array}{l}\text { Busco dedicarme a actividades fuera de la profesión (hobbies, } \\
\text { deportes, amistades, viajes) }\end{array}$ & 33,5 & 28,5 & $60,7^{*}$ \\
\hline $\begin{array}{l}\text { Pienso que no hay nada que hacer } \\
\text { Busco adaptarme con optimismo }\end{array}$ & 63,7 & 16,8 & 37,8 \\
\hline Me apoyo en mi vocación de servicio & 7,4 & 21,5 & $19^{*}$ \\
\hline Busco apoyo en mi grupo de trabajo & 6,6 & 16,8 & $71^{*}$ \\
\hline Busco agruparme y participar a través de actividades gremiales & 55,2 & 23,1 & $76,2^{*}$ \\
\hline
\end{tabular}

*Diferencia significativa entre las categorías de respuesta $p<0,05$. 
gremiales, predominando una actitud positiva ante los cambios. Setenta y un porciento de la muestra indica que frente a los cambios "frecuentemente" o "casi siempre" busca adaptarse con optimismo mientras que $19 \%$ piensa que no hay nada que hacer.

Las estrategias se asocian al género sólo respecto a la participación en actividades gremiales, siendo los hombres quienes participan con mayor frecuencia $(\mathrm{p}=0,05)$. La especialidad no se asocia a ninguna estrategia de afrontamiento examinada.

La edad presenta diferencias significativas en la estrategia: búsqueda de apoyo en la vocación de servicio y en el grupo de trabajo. Mientras los médicos de mayor edad tienden a apoyarse en la vocación de servicio $(\mathrm{p}<0,001)$, aquellos de menor edad lo harían en el grupo de trabajo ( $\mathrm{p}$ $=0,02$ ).

Finalmente, presentan significativamente mayor bienestar quienes tienden a adaptarse con optimismo ( $\mathrm{p}=0,0001)$ y afirman más frecuentemente optar por estrategias proactivas: buscan nuevas herramientas y enfoques para entender lo que pasa ( $\mathrm{p}=0,001)$, intentan mejorar sus capacidades con el estudio y perfeccionamiento $(\mathrm{p}=0,01)$, amplían sus actividades profesionales $(\mathrm{p}=0,01)$, buscan dedicarse a actividades fuera de la profesión $(\mathrm{p}=0,006)$, se apoyan en la vocación de servicio $(p=0,01)$ o en el grupo de trabajo $(\mathrm{p}=0,001)$ y buscan participar en actividades gremiales $(\mathrm{p}=0,007)$. Por el contrario presentan menor bienestar quienes tienden a pensar más frecuentemente que ante los cambios "no hay nada que hacer" $(\mathrm{p}=0,006)$.

\section{Discusión}

De acuerdo a los resultados obtenidos los médicos perciben cambios (moderados o profundos) en todos los ámbitos de la profesión examinados $y$ al menos $50 \%$ de ellos tienden a ser valorados en forma negativa. Se valoran negativamente con mayor frecuencia los cambios asociados a la disminución del reconocimiento, cohesión y autoregulación en la profesión.

Confirmamos que la percepción de cambios resulta ser un fenómeno más bien generalizado entre los médicos del mismo modo que la presencia de una actitud positiva, activa y basada en la profesión para hacerles frente $e^{8,17}$.
No se encontraron diferencias en la percepción de cambios según el género y sólo en algunos aspectos de acuerdo a la edad y especialidad del médico. Se observó que los médicos de mayor edad tienden más frecuentemente a percibir cambios y a valorarlos negativamente que los médicos de menor edad. Los médicos mayores de 51 años tienden a valorar negativamente con mayor frecuencia los cambios en los pacientes, el prestigio social y los nuevos requerimientos del sistema de salud.

Por su parte la especialidad o habitus más inmediato del médico define la relevancia de ciertos cambios por sobre otros. Por ejemplo, existe una menor percepción de cambios respecto a la presencia de médicos extranjeros entre los pediatras. Sin embargo, la especialidad no se asoció con ninguno de los aspectos de afrontamiento examinados.

En relación a la edad, los médicos mayores tienden a apoyarse en la vocación de servicio como estrategia de afrontamiento mientras aquellos más jóvenes se centran en el grupo de trabajo. Esto podría asociarse a la evolución de los médicos desde la cohesión grupal hacia un creciente desarrollo de su autonomía. Sin embargo, también puede sugerirse que el marco de identificación de los médicos se ha modificado. Esto sería consistente con la literatura que describe un cambio en el pacto tradicional entre médicos, empleadores y usuarios, incluyendo ahora nuevos actores que demandan por sus intereses y generando una necesidad de mayor cohesión entre los médicos.

El bienestar de los médicos se asoció a la percepción de cambios sólo en tres aspectos, mientras que la valoración positiva de los cambios y la mayor frecuencia con que se utilizan las estrategias de afrontamiento, se asocian con un mayor bienestar en la gran mayoría de los aspectos examinados.

Es posible señalar entonces que la valoración de los cambios más que la percepción de su existencia, afecta el bienestar de los médicos. Considerando que el uso de estrategias de afrontamiento afecta positivamente el bienestar, puede sugerirse que éstas constituyen una variable moderadora del impacto negativo de los cambios, facilitando la adaptación y bienestar de los médicos. Sin embargo, la naturaleza descriptiva de este estudio, no permite corroborar esta afirmación. Es decir, no sabemos si quienes se sienten mejor tienden a valorar positivamente los cambios y logran utilizar con mayor frecuencia las estrategias exploradas.

En general los datos disponibles describen 
por una parte los cambios percibidos y por otra el bienestar de los profesionales suponiendo una asociación entre ellos. Este estudio nos aporta información relevante al considerar el aspecto valorativo de los cambios percibidos en la profesión en relación a la adaptación de los médicos a los mismos en el contexto particular de nuestro país.

En esta línea es importante aludir a las características de la muestra y el contexto social en el cual se desarrolla el estudio. Si bien se consideró una muestra probabilística y representativa del universo, éste estuvo compuesto sólo por médicos inscritos en el Colegio Médico dada la imposibilidad de contar con otros registros conocidos del universo de médicos. Debido a las características del universo podrían estar sub-representados los grupos de mujeres y de médicos jóvenes. Finalmente, la muestra fue consultada cuando la reforma de salud estaba recientemente en marcha. Nuevos estudios podrán explorar la influencia de la reforma del sistema de salud en los profesionales así como precisar el papel mediador que sugerimos posee la valoración de los cambios y las estrategias para enfrentarlos en el bienestar de los médicos.

\section{Referencias}

1. Donelan K, Blendon RJ, Schoen C, Davis K, Binns K. The cost of health system change: public discontent in five nations. Health Affairs 1999; 18 (3): 206-16.

2. Mechanic D. Physician Discontent. Challenges and Opportunities. JAMA 2003; 290: 941-6.

3. Konrad TR, Williams ES, Hinzer M, McMurray J, Pathman DE, Gerritz M, et al. Measuring physician job satisfaction in a changing workplace and challengnig environment. Med Care 1999; 37: 1174-82.

4. Magee M, Hojat M. Impact of health care system on physician discontent. Journal of Community Health 2001; 26 (5): 357-65.

5. Haas JS, Cook EF, Puopolo AL, Burstin HR, Cleary PD, Brennan A. Is the Professional Satisfaction of General Internists Associated with Patient Satisfaction? Journal of General Internal Medicine 2000; 15 (2): 122-8.

6. Pathman DE, Konrad TR, Williams ES, Scheckler We, Linzer M, Douglas J. Physician job satisfaction, dissatisfaction, and turnover. J Fam Pract 2002; 51: 593-7.

7. Reames HR, Dunstone DC. Professional Satisfaction of Physicians. Arch Intern Med 1989; 149 (9): 1951-6.

8. Dunstone DC, Reames HR. Physician satisfaction revisited. Soc Sci Med 2001; 52 (6): 825-37.
9. Cossman JS, Street D. Policy, Autonomy and Physician Satisfaction. Journal of Health Care for the Poor and Underserved 2010; 21 (3): 898-912.

10. Janus K, Amelung VE, Gaitanides M, Schwartz FW. German physicians "on strike". Shedding light on the roots of physician dissatisfaction. Health policy 2007; 82: 357-65.

11. Wallace JE, Lemaire J. On physician well being. You will get by a little help from your friends. Social science \& Medicine 2007; 64: 2565-77.

12. Nettleton S, Burrows R, Wayy I. Regulating medical bodies? The consequences of the modernization of the NHS and the disembodiment of clinical knowledge. Sociology of health \&Illness 2008; 30: 0141-9889.

13. Harrison S. (2002) New labour, modernisation and the medical labour process,Journal of Social Policy , 31, 46585. En Nettleton, S.; Burrows, R.; Wavy, I. (2008) Op. cit.

14. Smith R. Why are doctors so unhappy? There are probably many causes, some of them deep. BJM 2001; 322: 1073-4.

15. Edwards N, Kornacki Mj, Silversin J. Unhappy doctors: what are the causes and what can be done? BMJ 2002; 324: 835-8.

16. Ham $\mathrm{CH}$, Alberti KG. The medical professionn, the public and the government. BMJ 2002; 324: 838-42.

17. Zuger A. Dissatisfaction with Medical Practice. N Engl J Med 2004; 350: 69-75.

18. Lewis CE, Prout DM, Chalmers EP, Leake B. How satisfying is the practice of internal medicine? A national survey. Ann Intern Med 1991; 114: 1-5.

19. Frank E, Mcmurray JE, Linzer M, Elon L. Career satisfaction of US women physicians: results from the Women Physicians' Health Study. Arch Intern Med 1999; 159: 1417-26.

20. Leigh JP, Kravitz RL, Schembri M, Samuels SJ, Mobley S. Physician career satisfaction across specialties. Arch Intern Med 2002; 162: 1577-84.

21. Petrozzi MC, Rosman HS, Nerenz DR, Young MJ. Clinical activities and satisfaction of general internists, cardiologists, and ophthalmologists. J Gen Intern Med 1992; 7: 363-5.

22. Hojat M, Gonnella JS, Erdmann JB, Veloski JJ, Louis DZ, Nasca TJ, RATTNER SL. Physicians' perceptions of the changing health care system: Comparisons by Gender and Specialties. J Community Health 2000; 25: 455-71.

23. OMS; South-East Asia, Assessment of subjective wellbeing. The subjective well-being inventory Regional Paper, SEARO, 1992, no 24.

24. Jiménez de la Jara J. Cambios en el entorno del trabajo médico. Rev Med Chile 2004; 132 (5): 637-42.

25. Horwitz N. El cambio de la práctica médica: Desafíos 
psicosociales para la profesión. Rev Med Chile 2004; 132 (6): 768-72.

26. Bascuñán ML. Cambios en la relación médico-paciente y nivel de satisfacción de los médicos. Rev Med Chile 2005; 133 (1): 11-6.

27. Jiménez de la Jara J P. Estrategias de los médicos para hacer frente a la crisis de la profesión. Rev Med Chile 2005; 133 (6): 707-12.
28. Horwitz N, Schiattino I, Bascuñán ML, Acuña J, Jiménez JP. Validación de una escala para medir el bienestar subje tivo de los médicos (BISUMED). Rev Med Chile 2010; 138: 1084-90.

29. Departamento de Economía, Universidad de Chile; Centro de Micro Datos. Informe final Encuesta Bienestar Subjetivo de los Médicos, Santiago, Septiembre 2007. 